\title{
Agaricomycetes gasteroides del bosque mesófilo de montaña, Estación Biológica Vasco de Quiroga, Michoacán, México
}

\author{
Gasteroid Agaricomycetes of the mountain cloud forest \\ from Vasco de Quiroga Biological Station, Michoacán, Mexico
}

\begin{abstract}
Mariela Salinas-Rodríguez", Martín Esqueda², Víctor Manuel Gómez-Reyes ${ }^{1}$, José Arnulfo Blanco-García1
${ }^{1}$ Facultad de Biología, Edificio "R", Ciudad Universitaria, Universidad Michoacana de San Nicolás de Hidalgo. Francisco J. Múgica s/n, Colonia Felicitas del Río, C.P. 58030, Morelia, Michoacán.

${ }^{2}$ Centro de Investigación en Alimentación y Desarrollo, A.C. Km 0.6 Carretera a La Victoria, C.P. 83304, Hermosillo, Sonora.
\end{abstract}

\section{RESUMEN}

Antecedentes: Los hongos gasteroides son importantes para el equilibrio de los ecosistemas, al ser degradadores de materia orgánica y presentar algunas especies micorrízicas. A pesar de su importancia, los estudios de este grupo son escasos y la mayoría se han enfocado a zonas áridas. En México, sólo un trabajo específico sobre la diversidad de hongos gasteroides en bosque mesófilo de montaña se ha publicado.

Objetivo: Contribuir al conocimiento de la diversidad de hongos gasteroides presentes en la Estación de Biológica Vasco de Quiroga, Uruapan, Michoacán.

Métodos: La recolecta de hongos gasteroides se realizó mediante recorridos exploratorios quincenales durante los meses de julio a noviembre del 2017. La determinación de las especies se realizó con base en características macro- y microscópicas con claves taxonómicas de bibliografía especializada.

Resultados y conclusiones: Se determinaron un total de diez especies pertenecientes a los géneros: Bovista (1), Geastrum (5), Lycoperdon (2) y Scleroderma (2). Destacan como nuevos registros para Michoacán y segundas citas para la micobiota de México: Bovista oblongispora var. oblongispora y Geastrum violaceum.

Palabras clave: taxonomía, corología, diversidad, Geastrum

\section{ABSTRACT}

Background: Gasteroid fungi are important in the balance of ecosystems, being decomposers of organic matter and some mycorrhizal species. Despite its importance, studies of this group are scarce and most of them have focused on arid areas. In Mexico only one specific work about gasteroid fungi diversity for the mountain cloud forest has been published. Objective: Contribute to the knowledge of the diversity of gasteroid fungi present in the Vasco de Quiroga Biological Station, Uruapan, Michoacán.

Methods: The collection of gasteroid fungi was gathered through exploratory survey, every two weeks during the months of July to November 2017. The determination of the species was made based on macro- and microscopic characteristics with taxonomic keys of specialized literature.

Results and conclusions: A total of 10 species belonging to genera Bovista (1), Geastrum (5), Lycoperdon (2), and Scleroderma (2) were determined. Bovista oblongispora var. oblongispora and Geastrum violaceum are new records for Michoacán and the second records for the Mexican mycobiota.

Keywords: taxonomy, chorology, diversity, Geastrum.

\section{ARTICLE HISTORY}

Received 01 July 2018 / Accepted 25 October 2018

On line 02 November 2018

\section{INTRODUCCIÓN}

El bosque mesófilo de montaña (BMM) se distingue por tener una vegetación con especies de afinidad boreal, tropical y endémicas; engloba una serie de asociaciones vegetales que se desarrollan en climas

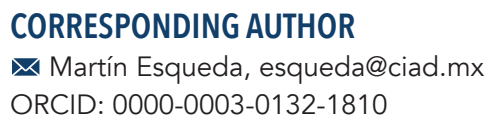

húmedos y cálidos (Rzedowski, 1978). En México, el BMM es el segundo tipo de vegetación con más registros de macromicetos, sólo superado por los bosques tropicales (Medel, 2007). Los hongos gasteroides son importantes en el equilibrio de los ecosistemas, ya que degradan la materia orgánica y coadyuvan en el reci- 
claje de nutrientes. Algunas especies pueden formar asociaciones micorrízicas con numerosos árboles y arbustos (Esqueda et al., 2011). Varios taxones se usan para tratar fracturas, dolor de oído, cicatrizante e incluso como cosméticos por diferentes tribus (Burk, 1983). Diversas especies son comestibles en etapa juvenil y en países como India, especímenes inmaduros de PheIlorinia se consideran un manjar (Sharma et al., 2015). Asimismo se han aislado compuestos antimicrobianos, antioxidantes, citotóxicos y antivirales de sus basidiomas (Al-Fatimi et al., 2005; Makarevich et al., 2012). A pesar de su importancia, los estudios sobre este grupo de hongos son escasos y la mayoría se han enfocado a zonas áridas.

Recientemente, Bautista-Hernández et al. (2018) registraron 20 especies de hongos gasteroides para el BMM de la Huasteca Alta Hidalguense y señalan que al igual que otros grupos de macromicetos, su diversidad es alta en este tipo de vegetación. En Michoacán, los estudios sobre este grupo de hongos son limitados, con 35 taxones registrados (Salinas-Rodríguez y Gómez-Reyes, 2015) y ninguno del BMM. Por lo que el presente trabajo tiene como objetivo contribuir al conocimiento sobre la diversidad de hongos gasteroides presentes en la Estación Biológica Vasco de Quiroga, Uruapan, Michoacán.

\section{MATERIALES Y MÉTODOS}

El presente estudio se realizó en la Estación Biológica Vasco de Quiroga, Uruapan, Michoacán, ubicada entre las coordenadas $19^{\circ} 15^{\prime} 00^{\prime \prime}-19^{\circ} 40^{\prime} 00^{\prime \prime}$ LN y $101^{\circ} 55^{\prime} 00^{\prime \prime}-$ $102^{\circ} 25^{\prime} 00^{\prime \prime} \mathrm{LO}$, con altitudes de 2100 a 2240 msnm. En el sitio se distinguen dos tipos de vegetación: BMM y bosque de pino-encino. El BMM está constituido por tres estratos: el arbóreo representado principalmente por especies perennifolias como Carpinus caroliniana Walter, el estrato arbustivo con especies de Montanoa Cerv., Cestrum L. y Solanum L. y finalmente el estrato herbáceo con taxones de Penstemon Schmidel., Adiantum L., Asplenium L., Pteridium Gled. ex Scop., Botrychium Sw. y Govenia Lindl. entre otras.

La recolecta de hongos gasteroides se realizó mediante recorridos exploratorios quincenales de julio a noviembre del 2017. La descripción de las características macro- y microscópicas se hicieron con base en Largent et al. (1984) y la determinación de los colores según Royal Botanic Garden Edinburgh (1969); además se realiza- ron cortes manuales con navaja del peridio y gleba, los cuales se hidrataron en hidróxido de potasio al $5 \%$ y tiñeron con rojo congo y/o solución de Melzer. La determinación taxonómica del material se realizó con base en bibliografía especializada (Sunhede, 1989; Pegler et al., 1995; Pérez-Silva et al., 1999; Bates et al., 2009; Bautista-Hernández et al., 2011; Guzmán et al., 2013). Las micrografías de esporas se tomaron en un microscopio electrónico de barrido (MEB) Joel JSM 6400; las muestras de gleba se montaron en un cilindro de carbón, se metalizaron con cobre por 15 min y finalmente se colocaron en el MEB. El orden sistemático se basó en Kirk et al. (2011), mientras que los nombres científicos y autores se basaron en la base de datos Index Fungorum (www.indexfungorum.org). El material estudiado se encuentra depositado en la Colección de Macromicetos del Herbario EBUM de la Facultad de Biología, Universidad Michoacana de San Nicolás de Hidalgo; abreviatura acorde con Index Herbariorum (Thiers, 2018).

\section{RESULTADOS}

Se determinaron 10 especies pertenecientes al Phyllum Basidiomycota, comprendidas en tres familias Agaricaceae (3), Geastraceae (5) y Sclerodermataceae (2). Bovista oblongispora var. oblongispora y Geastrum violaceum son nuevos registros para Michoacán y segundas citas para México.

\section{Descripción de las especies}

\section{Agaricomycetes, Agaricales, Agaricaceae}

Bovista oblongispora var. oblongispora (Lloyd) Bottomley, Bothalia 4(3): 580 (1948)

Figura 1

Basidioma 12-20 mm diámetro, globoso a subgloboso. Exoperidio blanco amarillento (6F), compuesto por pequeñas espinas. Endoperidio de blanco amarillento (6F) a marrón amarillento (8G). Gleba marrón amarillenta (8G) o marrón oscura (27). Subgleba de $0.5 \mathrm{~mm}$ de alto, color marrón amarillenta (8G) compacta, intemperizada. Cordones miceliales de color blanco de hasta $30 \mathrm{~mm}$ de largo. Capilicio tipo intermedio con hifas de 4.6-6 $\mu \mathrm{m}$ diámetro, pared gruesa y ramificadas. Basidios no observados. Esporas elipsoidales de $5.2-6 \times 3(-4) \mu \mathrm{m}$, verrugosas, con pedicelos caducos de 0.5-1.6 $\mu \mathrm{m}$ de largo. 
Material estudiado: Michoacán: Uruapan, Estación Biológica Vasco de Quiroga. M. Salinas 322, julio 7, 2017 (EBUM 29037). M. Salinas 324, julio 20, 2017 (EBUM 29038). M. Salinas 335, agosto 17, 2017 (EBUM 29039). Hábitat: Terrícola, en bosque mesófilo de montaña, fructificación de agosto a octubre.

Distribución: En México sólo se conocía de Veracruz (Bautista-Hernández et al., 2011). En esta ocasión se cita por primera vez para Michoacán y segundo registro para la micobiota de México.

Lycoperdon molle Pers., Syn. Meth. Fung. (Göttingen) 1: 150 (1801)

Figura 2

Basidioma 20-45 × 10-45 mm, subgloboso, piriforme u ovoide; peridio de $0.5 \mathrm{~mm}$ de grosor no separable de la gleba, color marrón grisáceo (26) en fresco a ma- rrón amarillento al secar (27); exoperidio conformado por pequeñas espinas simples y cortas, y verrugas, con ostiolo. Gleba blanca tornándose marrón oliváceo al madurar (33). Subgleba celular, color marrón claro (28). Capilicio con hifas de (2.5-)3.7-4.3 $\mu$ m diámetro, pared gruesa, no ramificadas. Basidios no observados. Esporas globosas a subglobosas de 5-6(-6.4) $\mu \mathrm{m}$ diámetro incluyendo la ornamentación, verrugosas, con pedicelos caducos de (9.5-)8.1-25.5(-28.8) $\mu \mathrm{m}$.

Material estudiado: Michoacán: Uruapan, Estación Biológica Vasco de Quiroga. M. Salinas 336, julio 20, 2017 (EBUM 29040). M. Salinas 350, agosto 4, 2017 (EBUM 29041). M. Salinas 372, septiembre 8, 2017 (EBUM 29042). M. Salinas 391, septiembre 22, 2017 (EBUM 29043). M. Salinas 405, octubre 7, 2017 (EBUM 29044). M. Salinas 415, octubre 22, 2017 (EBUM 29045). M. Salinas 420, noviembre 18, 2017 (EBUM 29046).
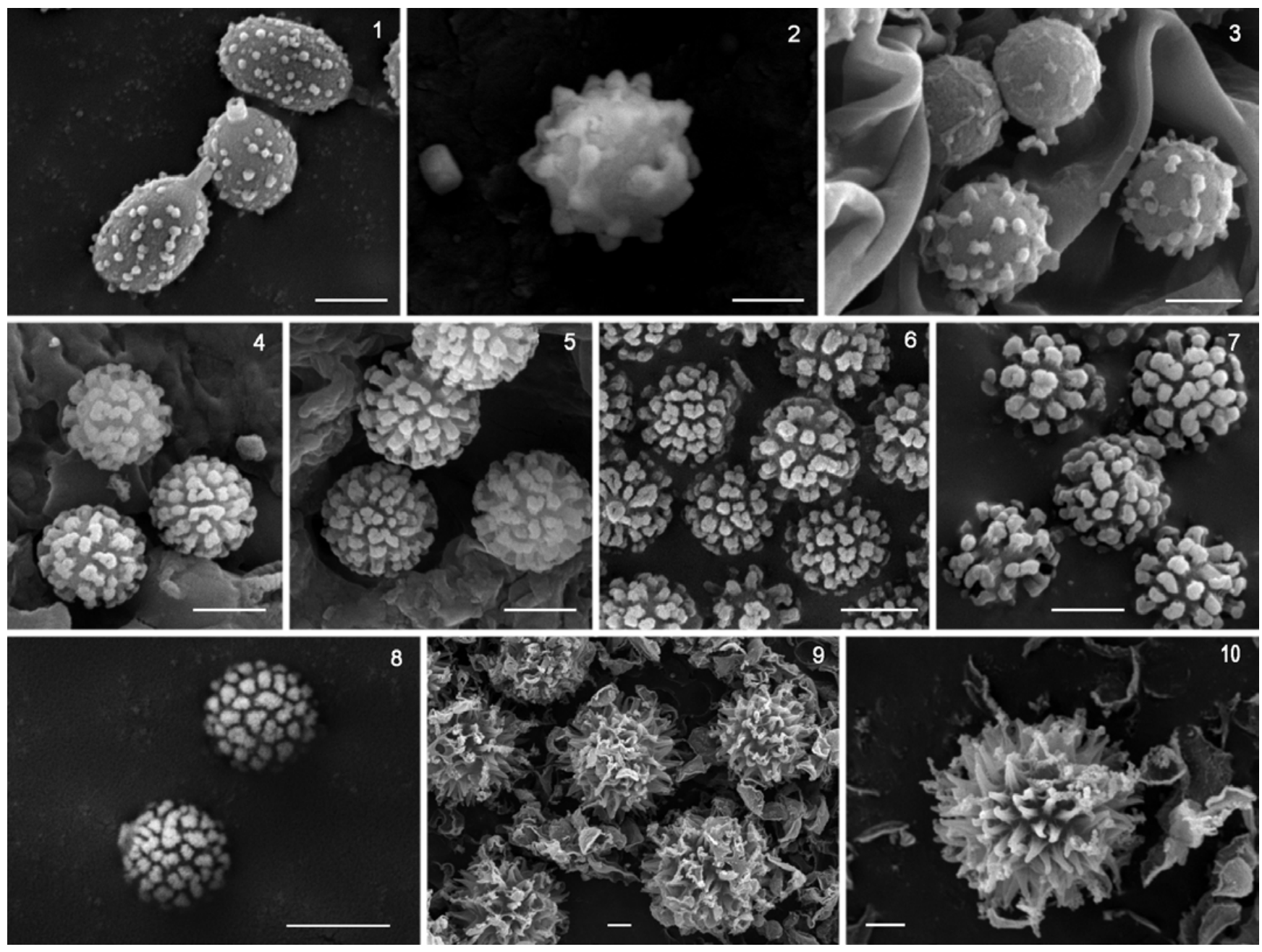

FIGURAS 1-10. Ornamentación esporal bajo microscopía electrónica de barrido. 1: Bovista oblongispora var. oblongispora. 2: Lycoperdon mole. 3: Lycoperdon perlatum. 4: Geastrum fimbriatum. 5: Geastrum lageniforme. 6: Geastrum saccatum. 7: Geastrum triplex. 8: Geastrum violaceum. 9: Scleroderma areolatum. 10: Scleroderma verrucosum. Escala $=2 \mu \mathrm{m}$. 
Hábitat: Terrícola, bosque mesófilo de montaña, fructificación de julio a noviembre.

Distribución: Registrada para Ciudad de México, Estado de México, Guerrero, Hidalgo (Calderón-Villagómez y Pérez-Silva, 1989) y Michoacán (Salinas-Rodríguez y Gómez-Reyes, 2015).

Lycoperdon perlatum Pers., Observ. Mycol. (Lipsiae) 1: 4 (1796)

Figura 3

Basidioma $37 \times 38-60 \mathrm{~mm}$, subgloboso, piriforme a ovoide, blanco a blanco amarillento (4D) en fresco, no cambia de color por fricción, blanco a marrón amariIlento al secar (27), superficie seca; exoperidio conformado por verrugas persistentes y espinas caducas de hasta $0.5 \mathrm{~mm}$ de longitud, con ostiolo. Gleba blanca; subgleba de $26 \times 10 \mathrm{~mm}$. Cordones miceliales blanquecinos. Peridio no separable de la gleba de $0.5 \mathrm{~mm}$ de grosor. Capilicio con hifas de (1.8-)3.4-4.2 $\mu \mathrm{m}$ diámetro, pared gruesa, ocasionalmente ramificada. Basidios no observados. Esporas globosas de 3.5-4.4 $\mu \mathrm{m}$, verrugosas.

Material estudiado: Michoacán: Uruapan, Estación Biológica Vasco de Quiroga. M. Salinas 326, julio 20, 2017 (EBUM 29047).

Hábitat: Terrícola, bosque mesófilo de montaña, fructificación en julio.

Distribución: Registrada para Chiapas, Chihuahua, Ciudad de México, Durango, Guanajuato, Guerrero, Hidalgo, Jalisco, Michoacán, Morelos, Nuevo León, Oaxaca, Puebla, San Luis Potosí, Sonora, Veracruz y Zacatecas (Varela y Cifuentes, 1979; Calderón-Villagómez y Pérez-Silva, 1989; Esqueda et al., 2000).

\section{Geastrales, Geastraceae}

Geastrum fimbriatum Fr., Syst. Mycol. (Lundae) 3(1): 16 (1829)

Figura 4

Basidioma 40-50 mm diámetro, globoso cuando joven. Al madurar exoperidio no higroscópico, esteliforme con 6 a 7 lacinias marrón claro (28) a marrón rojizo (19), curvadas involutas. Endoperidio globoso de 17-20 mm diámetro, sésil, marrón grisáceo (33), con superficie evidentemente granulosa. Peristoma más o menos cónico, finamente fibriloso, no delimitado. Gleba color morrón oscuro (26). Capilicio con hifas de 8-10 $\mu \mathrm{m}$ diámetro, pared delgada a gruesa, en ocasiones ramificadas. Basidios no observados. Esporas globosas de 3-4.5 $\mu$ m diámetro, equinadas, de color marrón oscuras en masa (16).

Material estudiado: Michoacán: Uruapan, Estación Biológica Vasco de Quiroga. M. Salinas 377, septiembre 8, 2017 (EBUM 29048). M. Salinas 393, septiembre 22, 2017 (EBUM 29049). M. Salinas 410, 411, 412, octubre 7, 2017 (EBUM 29050, 29051, 29052). M. Salinas 419, octubre 22, 2017 (EBUM 29053). M. Salinas 429, noviembre 18, 2017 (EBUM 29054).

Hábitat: Inicialmente semihipogeo, posteriormente epigeo, terrícola, en bosque mesófilo de montaña, fructificación de septiembre a noviembre. En regiones prioritarias para la conservación de la biodiversidad en Sonora, se ha observado en matorral tropical espinoso y bosque tropical caducifolio (Esqueda et al., 2003).

Distribución: Registrada para Campeche, Chiapas, Ciudad de México, Durango, Estado de México, Hidalgo, Jalisco, Michoacán, Morelos, Nayarit, Nuevo León, Puebla, Sonora, Veracruz y Zacatecas (Pérez- Silva et al., 1999; Esqueda et al., 2003; Herrera et al., 2005).

Geastrum lageniforme Vittad., Monogr. Lycoperd.: 16 (1842)

Figura 5

Basidioma 55-98 mm diámetro, globoso cuando joven. Al madurar exoperidio no higroscópico, esteliforme con 8 a 10 lacinias acuminadas. Endoperidio ovoide de 22-60 mm diámetro, color marrón claro (28) a marrón grisáceo (33), con ostiolo. Peristoma fimbriado, delimitado, color beige. Capilicio con hifas de (3.1-)7.2-10 $\mu \mathrm{m}$ diámetro, pared gruesa Basidios no observados. Esporas globosas de 4.6-5.5 $\mu \mathrm{m}$ diámetro, incluyendo ornamentación, equinadas, episporio grueso.

Material estudiado: Michoacán: Uruapan, Estación Biológica Vasco de Quiroga. M. Salinas 367, septiembre 8, 2017 (EBUM 20955). M. Salinas 378, 379, 380, septiembre 22, 2017 (EBUM 29056, 29057, 29058). M. Salinas 394, octubre 7, 2017 (EBUM 29059). M. Salinas 395, 396, 400, 401, 413, 416, octubre 7, 2017 (EBUM 29060, 29061, 29062, 29063, 29064, 29065).

Hábitat: Inicialmente hipogeo haciéndose epigeo al madurar, terrícola, en bosque mesófilo de montaña, fructificación de septiembre a octubre. En otras localidades se ha observado en bosque de Abies religiosa, encino, encino-pino, pino-encino, pino y cafetales (Pérez-Silva et al., 1999). 
Distribución: Se ha citado para Campeche, Ciudad de México, Estado de México, Guerrero, Hidalgo, Michoacán, Morelos, Nayarit, Oaxaca y Sonora (Pérez-Silva et al., 1999; Esqueda et al, 2003; Herrera et al., 2005).

Geastrum saccatum Fr., Syst. Mycol. (Lundae) 3(1): 16 (1829)

Figura 6

Basidioma 12-33 mm diámetro, globoso a saculiforme; peridio de $2 \mathrm{~mm}$ de grosor, agrietado, color marrón amarillento (12); cordones miceliales de hasta $125 \mathrm{~mm}$ de largo, con ostiolo. Exoperidio esteliforme al madurar con 6 a 8 lacinias, estriadas más o menos recurvadas hacia abajo, no higroscópicas, color marrón claro (28) a marrón rojizo (19). Endoperidio globoso de 14-26 mm diámetro, color marrón grisáceo (33). Peristoma cónico, finamente fimbriado y bien delimitado. Gleba color marrón oscuro (16). Capa micelial marrón claro al secar (32), con hifas septadas y fibuladas, de hialinas a color marrón oscuro (16), 5.4-7.0 $\mu \mathrm{m}$ diámetro. Capilicio con hifas de 3.2-3.5 $\mu \mathrm{m}$ diámetro, pared delgada a gruesa, ocasionalmente ramificada. Basidios no observados. Esporas globosas a subglobosas de 3.2-3.6 $\mu \mathrm{m}$ diámetro, fuertemente equinadas, episporio grueso.

Material estudiado: Michoacán: Uruapan, Estación Biológica Vasco de Quiroga. M. Salinas 354, agosto 4, 2017 (EBUM 29066). M. Salinas 376, septiembre 8, 2017 (EBUM 29067). M. Salinas 392, septiembre 22, 2017 (EBUM 29068). M. Salinas 408, octubre 7, 2017 (EBUM 29069). M. Salinas 417, octubre 22, 2017 (EBUM 29070).

Hábitat: Terrícola, en bosque mesófilo de montaña, bosque de pino-encino, fructificación de agosto a octubre.

Distribución: Se ha registrado para Campeche, Chihuahua, Ciudad de México, Durango, Guerrero, Hidalgo, Jalisco, Michoacán, Nayarit, Nuevo León, Puebla, Sonora, Tlaxcala y Veracruz (Pérez-Silva et al., 1999; Esqueda et al, 2003; Herrera et al., 2005; Moreno et al., 2010).

Geastrum triplex Jungh. Tijdschr. Nat. Gesch. Physiol. 7: 287 (1840)

Figura 7

Basidioma 35-72 mm diámetro, al principio ovoide de $37 \mathrm{~mm}$ diámetro, al madurar exoperidio esteliforme con 5 a 8 lacinias no higroscópicas de color marrón claro (29) a marrón rojizo (19); mesoperidio bien desa- rrollado en forma de collar de $19-30 \mathrm{~mm}$ de alto. Endoperidio ovoide, marrón claro (17) a marrón grisáceo oscuro (26), con ostiolo. Peristoma fimbriado delimitado. Capilicio con hifas de (2.6-)3.1-7 um diámetro, pared gruesa. Basidios no observados. Esporas globosas de (3.6-)4.5-5 $\mu \mathrm{m}$ diámetro, fuertemente equinadas, episporio grueso.

Material estudiado: Michoacán: Uruapan, Estación Biológica Vasco de Quiroga. M. Salinas 368, 369, septiembre 8, 2017 (EBUM 29071, 29072). M. Salinas 381, 382, 383, septiembre 22, 2017 (EBUM 29073, 29074, 29075). M. Salinas 397, 398, 399, octubre 7, 2017 (EBUM 29076, 29077, 29078).

Hábitat: Al principio hipogeo y al madurar epigeo, terrícola, en bosque mesófilo de montaña, fructificación de septiembre a octubre.

Distribución: Registrado para Chiapas, Chihuahua, Ciudad de México, Estado de México, Guerrero, Hidalgo, Jalisco, Michoacán, Oaxaca, Sonora y Veracruz (Varela y Cifuentes, 1979; Pérez-Silva et al., 1999; Moreno et al., 2010).

Geastrum cf. violaceum Rick Brotéria 5: 26, 1906 Figura 8

Basidioma 14-25 mm diámetro, al principio ovoide acuminado, al madurar exoperidio no higroscópico esteliforme con 7 lacinias, de color rosa pálido (76) curvado involutas. Endoperidio ovoide de 5-12 mm diámetro, color marrón grisáceo claro (33), con ostiolo. Peristoma fimbriado, ligeramente plegado. Superficie del endoperidio c on micoesclereidas de 4.8-9.9 $\times$ 29.6-31.8 $\mu \mathrm{m}$, pared gruesa. Capa micelial marrón anaranjado (12) a marrón grisáceo (33) al secar, compuesta por hifas que pueden presentar fíbulas de 5-6 $\mu \mathrm{m}$ diámetro, pared delgada, color marrón oliva (27). Capa seudoparenquimatosa formada por células globosas hialinas de pared delgada. Capilicio con hifas de 2-4.5(-5.0) $\mu \mathrm{m}$ diámetro, pared gruesa, marrón (16), sin fíbulas. Basidios no observados. Esporas globosas de (2.2-)2.4-3.1 um diámetro, equinadas.

Material estudiado: Michoacán: Uruapan, Estación Biológica Vasco de Quiroga. M. Salinas 349, agosto 4, 2017 (EBUM 29079). M. Salinas 384, 385, 386, septiembre 22, 2017 (EBUM 29080, 29081, 29082). M. Salinas 402, octubre 7, 2017 (EBUM 29083).

Hábitat: Al principio hipogeo, posteriormente epigeo, humícola, en bosque mesófilo de montaña, fructificación de agosto a octubre. 
Distribución: Bautista-Hernández et al. (2015) registraron por primera vez este taxón para México de Jalisco, Querétaro, San Luis Potosí y Tabasco. Se cita por primera vez para Michoacán.

\section{Boletales, Sclerodermataceae}

Scleroderma areolatum Ehrenb., Sylv. Mycol. Berol. (Berlin): 27 (1818)

Figura 9

Basidioma 12-26 mm diámetro, globoso a subgloboso, color marrón amarillento (12) tornándose rojo pálido (41) con la fricción, blanco amarillento al secar (9H); peridio no separable de la gleba, 0.5-1 mm de grosor. Exoperidio con escamas planas, irregulares, negruzcas (16), con ostiolo. Gleba color marrón oscuro (36). Cordones miceliales amarillentos (4D). Capilicio con hifas de 3.4-4 $\mu \mathrm{m}$ diámetro, pared delgada, ramificadas. Basidios no observados. Esporas globosas de 13-15(-18) $\mu \mathrm{m}$ diámetro incluyendo la ornamentación, fuertemente equinadas, con espinas de 1.5-2.7 $\mu \mathrm{m}$ de largo.

Material estudiado: Michoacán: Uruapan, Estación Biológica Vasco de Quiroga. M. Salinas 333, 338, 339, julio 20, 2017 (EBUM 29084, 29085, 29086). M. Salinas 345, 346, agosto 04, 2017 (EBUM 29087, 29088). M. Salinas 356, 357, agosto 17, 2017 (EBUM 29089, 29090). M. Salinas 370, septiembre 8, 2017 (EBUM 29091). M. Salinas 406, octubre 7, 2017 (EBUM 29092).

Hábitat: Terrícola, en bosque mesófilo de montaña, fructificación de julio a octubre.

Distribución: Registrada para Aguascalientes, Chiapas, Chihuahua, Ciudad de México, Durango, Guerrero, Hidalgo, Jalisco, Michoacán, Morelos, Nuevo león, Oaxaca, Puebla, Quintana Roo, Sonora, Tlaxcala y Veracruz (Guzmán et al., 1997, 2013; Esqueda et al., 2000).

Scleroderma verrucosum (Bull.) Pers., Syn. Meth. Fung. (Göttingen) 1: 154 (1801)

Figura 10

Basidioma 13-40 mm diámetro, globoso a subgloboso, color marrón amarillento (52) a marrón rojizo (12) en fresco, tornándose rojo pálido (42) por fricción, blanco amarillento al secar (5E); peridio de 0.5-1 mm de grosor. Exoperidio con escamas planas e irregulares, color marrón oscuro (18); la superficie del endoperidio con agrietamiento irregular apical. Sésil o con pseudoestipite. Cordones miceliales amarillentos (4D) de 10-25 mm largo. Gleba color marrón oscuro (16). Capilicio con hifas de 2-3.6 $\mu \mathrm{m}$ diámetro, pared delgada, ramificadas. Basidios no observados. Esporas globosas de 11.4-14(-15) $\mu \mathrm{m}$ incluyendo ornamentación, fuertemente equinadas, con espinas de (0.6-)1.2-2 $\mu \mathrm{m}$ de largo.

Material estudiado: Michoacán: Uruapan, Estación Biológica Vasco de Quiroga. M. Salinas 328, 329, 330, julio 20, 2017 (EBUM 29093, 29094, 29095). M. Salinas 348, 352, 353, 354, agosto 04, 2017 (EBUM 29096, 29097, 29098, 29099). M. Salinas 358, 359, 360, 361, 362, agosto 17, 2017 (EBUM 29100, 29101, 29102, 29103, 29104). M. Salinas 374, septiembre 8, 2017 (EBUM 29105). M. Salinas 388, septiembre 22, 2017 (EBUM 29106). M. Salinas 407, 408, octubre 7, 2017 (EBUM 29107, 29108). M. Salinas 409, octubre 21, 2017 (EBUM 29109).

Hábitat: Terrícola, bosque mesófilo de montaña, fructificación de julio a octubre.

Distribución: Taxón citado para Aguascalientes, Chiapas, Chihuahua, Estado de México, Guerrero, Hidalgo, Jalisco, Michoacán, Morelos, Nuevo León, Oaxaca, Puebla, San Luis Potosí, Sonora y Veracruz (Varela y Cifuentes, 1979; Esqueda et al., 2000; Guzmán et al., 1997, 2013).

\section{DISCUSIÓN}

De las 10 especies determinadas en el presente trabajo, dos son nuevos registros para Michoacán. Bovista oblongispora var. oblongispora es una especie que se caracteriza por presentar esporas elipsoidales, verrugosas y capilicio no porado. Esta especie es similar a B. oblongispora var. longispora, la cual se diferencia por sus hifas poradas del capilicio. El conocimiento sobre su distribución estaba limitado a Veracruz en bosque mesófilo de montaña (Bautista-Hernández et al., 2011) y en este trabajo se cita por primera vez para Michoacán. Geastrum violaceum es una especie que se caracteriza principalmente por el color rosa, rojo a violeta pálido, además de la presencia de un peristoma fimbriado ligeramente plegado; esta especie se registró por primera vez para el país en bosque tropical caducifolio (Bautista-Hernández et al., 2015). Sin embargo, en el estudio realizado por Campi et al. (2013), mencionan que esta especie suele crecer cerca de Fabaceae y Lauraceae en selva subtropical lluviosa. Aunque la Estación Biológica Vasco de Quiroga incluye zonas destinadas al cultivo de aguacate (Lauraceae), aún falta por conocer la influencia de la distribución y plasticidad morfológica de G. violaceum. Para ello, 
los especímenes de Michoacán se compararán a nivel molecular con los de Jalisco y Tabasco.

\section{LITERATURA CITADA}

Al-Fatimi, M., M. Wurster, H. Kreisel, U. Lindequist, 2005. Antimicrobial, cytotoxic and antioxidant activity of selected basidiomycetes from Yemen. Pharmazie 60: 776-780.

Bates, S.T., R.W. Roberson, D.E. Desjardin, 2009. Arizona gasteroid fungi I: Lycoperdaceae (Agaricales, Basidiomycota). Fungal Diversity 37: 153-207.

Bautista-Hernández, S., T. Herrera, E. Aguirre-Acosta, M. Esqueda. 2011. Contribution to the taxonomy of Bovista in Mexico. Mycotaxon 118: 27-46.

Bautista-Hernández, S., E. Aguirre-Acosta, T. Raymundo, T. Herrera, J. Cifuentes, S. Capello-García, R. Valenzuela. 2015. Geastrum violaceum, registro nuevo para México. Revista Mexicana de Biodiversidad 86: 569-572.

Bautista-Hernández, S., T. Raymundo, E. Aguirre-Acosta, M. Contreras-Pacheco, L. Romero-Bautista, R. Valenzuela, 2018. Agaricomycetes gasteroides del bosque mesófilo de montaña de la Huasteca Alta Hidalguense, México. Acta Botánica Mexicana 123: 21-36.

Burk, W.R., 1983. Puffball usages among North American indians. Journal of Ethnobiology 3: 55-62.

Calderón-Villagómez, A., E. Pérez-Silva, 1989. Consideraciones taxonómicas y nuevos registros de algunas especies del género Lycoperdon (Gasteromycetes) en México. Anales del Instituto de Biología Universidad Nacional Autónoma de México Serie Botánica 59: 1-30.

Campi, M., B. Madrignac, A. Flecha, A. Ortellado, M.R. Gullón, 2013. Geastrum violaceum Rick (Geastraceae, Basidiomycota), nuevo registro para Paraguay. Reportes Científicos FACEN 4(2): 15-18.

Esqueda, M., E. Pérez Silva, T. Herrera, M. Coronado, A. Estrada-Torres, 2000. Composición de gasteromicetos en un gradiente de vegetación de Sonora, México. Anales del Instituto de Biología Universidad Nacional Autónoma de México Serie Botánica 71: 39-62.

Esqueda, M., T. Herrera, E. Perez-Silva, A. Sánchez, 2003. Distribution of Geastrum species from some priority regions for conservation of biodiversity of Sonora, Mexico. Mycotaxon 87: 445-456.

Esqueda, M., A. Sánchez, M.L. Coronado, A. Gutiérrez, M. Lizárraga, R. Valenzuela, 2011. Nuevos registros de hongos gasteroides en la Reserva de Biosfera Sierra de Álamos-Río Cuchujaqui. Revista Mexicana de Micología 34: 43-51.

Guzmán, G., A. Cortés-Pérez, L. Guzmán-Dávalos, F. Ramírez-GuiIlén, M.R. Sánchez-Jácome, 2013. An emendation of Scleroderma, new records and review of the known species in Mexico. Revista Mexicana de Biodiversidad 84: S173-S191.
Guzmán, G., F. Tapia, P. Navarro, F. Ramírez-Guillén, 1997. Sclerodermataceous fungi (Basidiomycota, Gasteromycetideae) from Mexico and new reports. Documents Mycologiques 26: 53-58.

Herrera, T., E. Pérez-Silva, M. Esqueda, V.H. Valenzuela. 2005. Algunos gasteromicetos de Calakmul, Campeche, México. Revista Mexicana de Micología 21: 23-27.

Kirk, P.M., P.F. Cannon, D.W. Minter, J.A. Stalpers, 2011. Ainsworth and Bisby's Dictionary of the Fungi. CAB International, Wallingford.

Largent, D., D. Johnson, R. Watling, 1984. How to Identify Mushrooms to Genus III: Microscopic Features. Mad River Press, Eureka.

Makarevich, E.V., Z.B. Ibragimova, T.A. Kosogova, O.G. Kurskaya, O. Mazurkov, T.N. Ilyicheva, T.V. Teplyakova, N.A. Mazurkova, 2012. Immunogenic and protective properties of extracts from higher fungi from a group of orders Gasteromycete. Microbiology 38: 68-72.

Medel, R., 2007. Ascomycetes citados de México IV: 1996-2006. Revista Mexicana de Micología 25: 69-76.

Moreno, G., M. Lizárraga, M. Esqueda, M.L. Coronado, 2010. Contribution to de study of gasteroid and secotioid fungi of Chihuahua, Mexico. Mycotaxon 112: 291-325.

Pegler, D.N., T. Laessoe, B.M. Spooner, 1995. British puffballs, earthstars and stinkhorns; an account of the British Gasteroid fungi. Royal Botanic Gardens, Kew.

Pérez-Silva, E., T. Herrera, M. Esqueda, 1999. Species of Geastrum (Basidiomycotina: Geastraceae) from Mexico. Revista Mexicana de Micologia 15: 89-104.

Royal Botanic Garden Edinburgh, 1969. Flora of British fungi: color identification chart. Royal Botanic Garden, Edinburgh.

Rzedowski, J., 1978. Vegetación de México. Limusa, México.

Salinas-Rodríguez, M., V.M. Gómez-Reyes, 2015. Gasteromicetos del Estribo Grande y Estribo Chico del Municipio de Pátzcuaro, Michoacán. Biológicas 17(2): 10-14.

Sharma, V.P., M. Singh, S. Kumar, S. Kamal, R. Singh, 2015. Phylogene and physiology of Phellorinia spp.: a delicacy of Indian desert. International Research Journal of Natural and Applied Sciences 2(4): 1-17.

Sunhede, S., 1989. Geastraceae (Basidiomycotina). Morphology, ecology, and systematics with special emphasis on the North European species. Synopsis Fungorum 1. Fungiflora, Gronlands Grafiske A/S, Oslo.

Thiers, B. 2018. Index Herbariorum: A global directory of public herbaria and associated staff. New York Botanical Garden's Virtual Herbarium. http://sweetgum.nybg.org/ih/

Varela, L., J. Cifuentes, 1979. Distribución de algunos macromicetos en el norte del estado de Hidalgo. Boletín de la Sociedad Mexicana de Micología 13: 75-88. 